This is a post-peer-review, pre-copyedit version of a conference paper published in EuCoMeS 2018. EuCoMeS 2018. Mechanisms and Machine Science 59 : 45-52 (2019). The final authenticated version is available online at: http://dx.doi.org//10.1007/978-3-319-98020-1_6

\title{
Mechatronic model based jerk optimization in servodrives with compliant load
}

\author{
I. Ansoategui ${ }^{1}$ and F.J. Campa ${ }^{2}$ \\ ${ }^{1}$ University of the Basque Country UPV/EHU, Spain, e-mail: \\ igor.ansoategui@ehu.eus \\ 2 University of the Basque Country UPV/EHU, Spain, e-mail: fran.campa@ehu.eus
}

Abstract.

The aim of this work is to develop a mechatronic model for servodrives that move a compliant load, which is the case of large heavy machine tools. The model integrates the dynamics of the motor, transmission chain and compliant load as well as the dynamics of the control and is used to calculate the maximum overshoot in the load when it reaches the commanded final position as a function of the feed speed and the programmed jerk in the velocity profile. This overshoot must be, in applications as machining, always minimized. The result is a graph that indicates the region of safe values of jerk, where a given overshoot is never surpassed, but also there are several regions where much higher jerk values can be programmed with minimal overshoot. The location of these areas depending on the feed speed and the load natural frequency has been shown for a square sine profile. These optimal values of the jerk have been experimentally validated.

Key words: mechatronics, servodrives, machine tools, jerk optimization, path planning.

\section{Introduction}

One of the main problems that the designers of drives for positioning heavy loads is the compliance of the load itself, due to the lack of stiffness. The result is the onset of vibrations in the load due to the inertial forces, and also the problem of finding the optimal control gains and path planning in order to avoid them. The result is usually having to employ very low control gains and conservative path planning, with low acceleration and jerk. An example is the industry of heavy machine tools, where having to move heavy rams and columns up to 5 meters length and a natural frequency of $10-15 \mathrm{~Hz}$ is not uncommon. The use of a mechatronic model integrating the dynamics of the drive, transmission and load together with the control can help improving the design of such systems but also determining the optimal jerk and acceleration in the path planning. 
Elemental mechatronic models for servodrives consider just inertial models of the system [4]. These models, provide and initial estimation of the behaviour and frequently results precise enough. However, if the load inertia is very high, the flexibility of the transmission must be taken into account, being the most common approach the use of a 2 degrees of freedom (dof) model of the drivetransmission-load system as the ones proposed by Dequidt [5], Altintas [6] or Caracciolo [7]. However, it may well happen that the load is also compliant, as in the case of heavy machine tools, so, to have a precise model of the mechanical system, a 3 dof model must be used, as in [8-10]. In these cases, the third dof is the position at the end of the load, which is the part or the machine element that must be moved and is compliant, see Fig. 1. This position is never measured nor considered in the position control due to accessibility problems. That means that the NC of the machine is just capable of controlling the position of the second dof and the relative displacement that happens between the second and third dofs is not avoidable. The result is the onset of vibration in the acceleration/decceleration and specially the appearance of an overshoot in the final position that can compromise the geometry of the part machined.

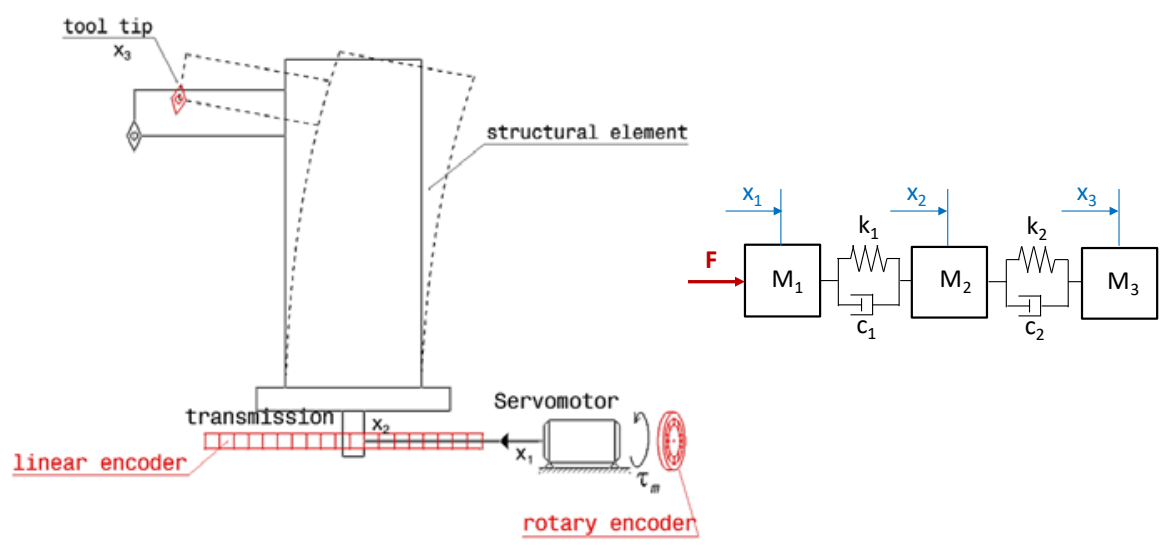

Fig. 1. Influence of the column flexibility on the tool error and degrees of freedom: $\mathrm{x}_{1}$, drive encoder position, $\mathrm{x}_{2}$, linear encoder position, $\mathrm{x}_{3}$, tool position. Corresponding 3 dof model.

Here, a 3 dof mechatronic model of a drive with a compliant load is presented and experimentally validated in a test bench. The model is then used to predict the optimal jerk and acceleration values to program in the path planning minimizing vibrations due to inertial forces and overshoot. Hence, the minimization of the vibration is reached without using external devices or modifying the control algorithm of the NC, as the use of open controls is not common in machine tool industry. 


\section{Mechatronic model of a 3 dof system}

In Fig. 1 the 3 dof lumped parameters model of the mechanical system drivetransmission-load, following the guidelines in [12], where $x_{1}$ is the position of the drive measured at the encoder, $x_{2}$ is the position measured at the linear encoder at the end of the transmission, and $x_{3}$ is the position at the end of the load. Masses $m_{1}, m_{2}, m_{3}$ represents the inertia of the drive, transmission and load respectively, $k_{1}$ and $k_{2}$ are the stiffness of the drive-transmission system and the load, and finally, $c_{1}$ and $c_{2}$ are the corresponding viscous damping. $F$ is the force equivalent to the motor torque. The equations of motion are:

$$
\left[\begin{array}{ccc}
m_{1} & 0 & 0 \\
0 & m_{2} & 0 \\
0 & 0 & m_{3}
\end{array}\right]\left\{\begin{array}{l}
\ddot{x}_{1} \\
\ddot{x}_{2} \\
\ddot{x}_{3}
\end{array}\right\}+\left[\begin{array}{ccc}
c_{1} & -c_{1} & 0 \\
-c_{1} & c_{1}+c_{2} & -c_{2} \\
0 & -c_{2} & c_{2}
\end{array}\right]\left\{\begin{array}{l}
\dot{x}_{1} \\
\dot{x}_{2} \\
\dot{x}_{3}
\end{array}\right\}+\left[\begin{array}{ccc}
k_{1} & -k_{1} & 0 \\
-k_{1} & k_{1}+k_{2} & -k_{2} \\
0 & -k_{2} & k_{2}
\end{array}\right]\left\{\begin{array}{l}
x_{1} \\
x_{2} \\
x_{3}
\end{array}\right\}=\left\{\begin{array}{l}
F \\
0 \\
0
\end{array}\right\}
$$

The equations of motion in time domain are converted to the Laplace domain, where the three degrees of freedom are related with the force by means of three transfer functions (TF): $T F_{1}$ relates $x_{1}$ with $F, T F_{2}$ relates $x_{2}$ with $x_{1}$ and $T F_{3}$ relates $\mathrm{x}_{3}$ with $\mathrm{x}_{2}$. These transfer functions are represented in a general form in Eq. 2, whose coefficients are shown in Table 1.

$$
T F_{i}=\frac{n_{4} s^{4}+n_{3} s^{3}+n_{2} s^{2}+n_{1} s+n_{0}}{d_{6} s^{6}+d_{5} s^{5}+d_{4} s^{4}+d_{3} s^{3}+d_{2} s^{2}+d_{1} s+d_{0}}
$$


Table 1. Numerator and denominator coefficients in the Transfer Functions.

\begin{tabular}{|c|c|c|c|}
\hline & $F T_{1}$ & $F T_{2}$ & $F T_{3}$ \\
\hline$n_{0}$ & $k_{1} k_{2}$ & $k_{1} k_{2}$ & $k_{2}$ \\
\hline$n_{1}$ & $c_{1} k_{2}+c_{2} k_{1}$ & $c_{1} k_{2}+c_{2} k_{1}$ & $c_{2}$ \\
\hline$n_{2}$ & $m_{3} k_{1}+\left(m_{2}-m_{3}\right) k_{2}+c_{1} c_{2}$ & $m_{3} k_{1}+c_{1} c_{2}$ & 0 \\
\hline$n_{3}$ & $m_{3} c_{1}+\left(m_{2}-m_{3}\right) c_{2}$ & $m_{3} c_{1}$ & 0 \\
\hline$n_{4}$ & $m_{2} m_{3}$ & 0 & 0 \\
\hline$d_{0}$ & 0 & $k_{1} k_{2}$ & $k_{2}$ \\
\hline$d_{1}$ & 0 & $c_{1} k_{2}+c_{2} k_{1}$ & $c_{2}$ \\
\hline$d_{2}$ & $\left(m_{1}+m_{2}-m_{3}\right) k_{1} k_{2}$ & $m_{3} k_{1}+\left(m_{2}-m_{3}\right) k_{2}+$ & $m_{3}$ \\
\hline$d_{3}$ & $\left(m_{1}+m_{2}-m_{3}\right) \cdot\left(c_{2} k_{1}+c_{1} k_{2}\right)$ & $m_{3} c_{1}+\left(m_{2}-m_{3}\right) c_{2}$ & 0 \\
\hline$d_{4}$ & $\begin{array}{c}\left(m_{1}+m_{2}\right) m_{3} k_{1}+\left(m_{2}-m_{3}\right) m_{1} l \\
+\left(m_{1}+m_{2}-m_{3}\right) c_{1} c_{2}\end{array}$ & $m_{2} m_{3}$ & 0 \\
\hline$d_{5}$ & $\left(m_{1}+m_{2}\right) m_{3} c_{1}+\left(m_{2}-m_{3}\right) m_{1}$ & 0 & 0 \\
\hline$d_{6}$ & $m_{1} m_{2} m_{3}$ & 0 & 0 \\
\hline
\end{tabular}

These transfer functions are integrated then in a mechatronic model programmed in Simulink, where the velocity and position feedback loops are represented, see Fig. 2. Note that $\mathrm{TF}_{3}$ is after the position feedback loop, as there is no direct measurement of $x_{3}$. The model has been developed thinking in a rotary drive whose rotation is then converted to a translation with a transmission factor $i(\mathrm{~m} / \mathrm{rad})$. Hence, $\mathrm{TF}_{1}$ must be derived to obtain the motor velocity $\omega_{m}$ to close the velocity feedback loop: $\omega_{m}=\dot{x}_{1} / i . F$ is related to the motor torque by $\tau_{m}=F \cdot i . \mathrm{TF}_{2}$ is also adapted to relate $\omega_{m}$ and $\mathrm{x}_{2}$. Finally, a simple viscousCoulomb friction model has been introduced and then identified experimentally.

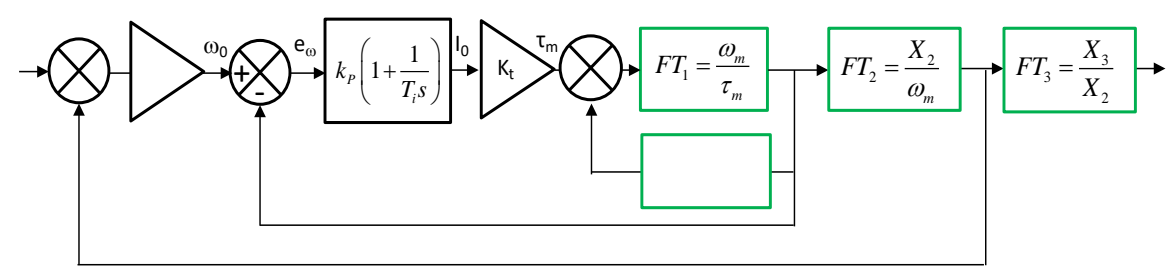

Fig.2. 3 dof mechatronic model of a servodrive with compliant load. 
Regarding the control, it is the usual cascade control used in NC machine tool with three cascaded feedback loops of position, velocity and current. The position control is Proportional, the velocity control is Proportional-Integral, and the current control is also Proportional-Integral, although it is not modelled since the loop closing period of this loop is much lower than the dynamics of the velocity and position loops, that is, the electrodynamics will be much faster than the dynamics of the mechanical parts.

\section{Test bench description}

A test bench has been set up, see Fig. 3, with a Fagor 42.30A FKM servodrive with rotary encoder and a nominal torque of 6,3 Nm, a ball screw Korta KBS-3210 with a diameter of $32 \mathrm{~mm}$ and a lead of $10 \mathrm{~mm}$, a linear encoder Heidenhain Ls 186 MI640 with a resolution of $0,5 \mu \mathrm{m}$. The NC is a Fagor 8035. On the table, the load is a mass of $30 \mathrm{~kg}$ over two steel plates with a thickness of $1,5 \mathrm{~mm}$ to introduce compliance. The position of the mass is externally measured using a linear interferometer HP 5529A.

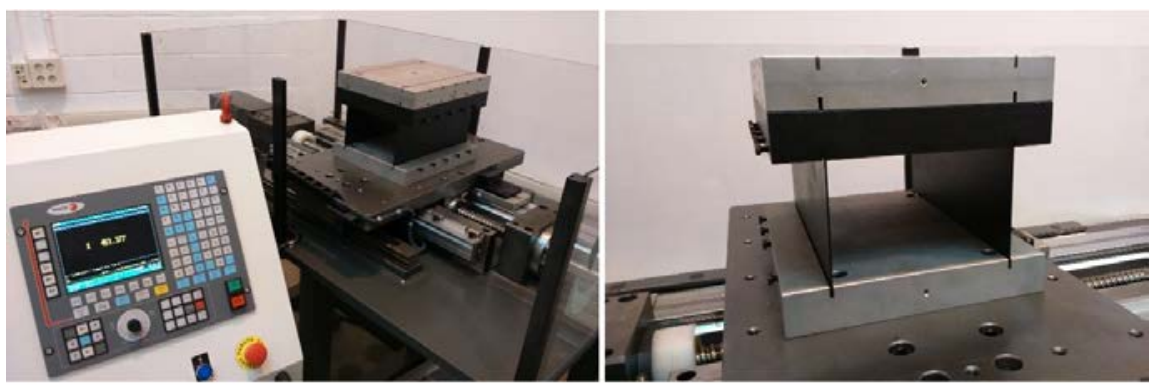

Fig. 3. Test bench: compliant load over a ball-screw drive.

After modelling all the elements of the system, the modal analysis reveals a first mode of $15,1 \mathrm{~Hz}$ due to the thin plates. This amplification is clearly seen in $\mathrm{TF}_{3}$ in Fig. 4a. Fig. 4b compares the Bode plot of the position closed loop transfer function ( $T F_{C P L}$ ), which relates $x_{2}$ with the position command $x_{0}$, with the product $\mathrm{TF}_{\mathrm{CPL}} \cdot \mathrm{TF} 3$, which relates the position command with $\mathrm{x}_{3}$. It can be seen how, as expected, although the closed loop is tuned with no resonance and a bandwidth of $8,9 \mathrm{~Hz}$, the addition of a compliant load results in a resonance near its natural frequency that will amplify harmonics of the motion nearby. The influence of the compliant mass mode also appears in $\mathrm{TF}_{1}$ and $\mathrm{TF}_{2}$. 

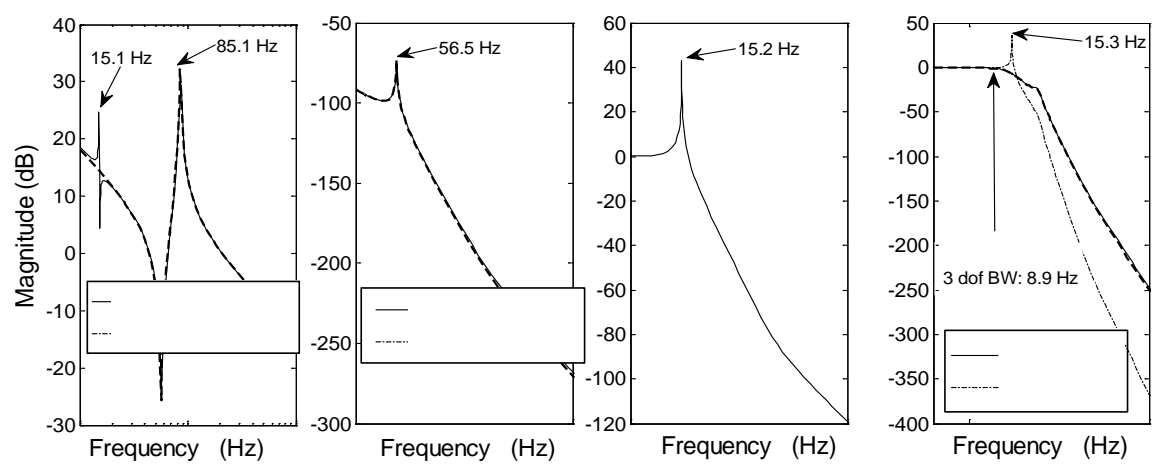

Fig. 4. $\mathrm{TF}_{1}, \mathrm{TF}_{2}, \mathrm{TF}_{3}$ and $\mathrm{TF}$ CPL bode diagrams.

\section{Experimental Validation}

Several tests have been made ranging displacements from 20 to $400 \mathrm{~mm}$ and feed speeds from 7 to $30 \mathrm{~m} / \mathrm{min}$ with a square sine velocity profile. For a feed speed of $7 \mathrm{~m} / \mathrm{min}$ and a displacement from the zero position to $100 \mathrm{~mm}$ and back to the zero, Fig. 6 compares the error in the mass position $x_{3}$, modelled and predicted, and the table position $x_{2}$, modelled and predicted also, whose difference is better seen in the zoom. Although there are deviations probably due to the damping estimation, the model matches the reality satisfactorily taken into account the simplifications done. It can be seen how there is a clear vibration that begins in the initial accelerations and also how when the final position is reached, near the 2 seconds, the position error in the mass reaches values up to $1 \mathrm{~mm}$. All the tests whose motion profile present harmonics near the load natural frequency present this problem. Also, the vibration affects the position of the ball screw table which oscillates up to $20 \mu \mathrm{m}$ as it can be seen in the zoom between $0 \mathrm{~s}$ and $0,9 \mathrm{~s}$. 


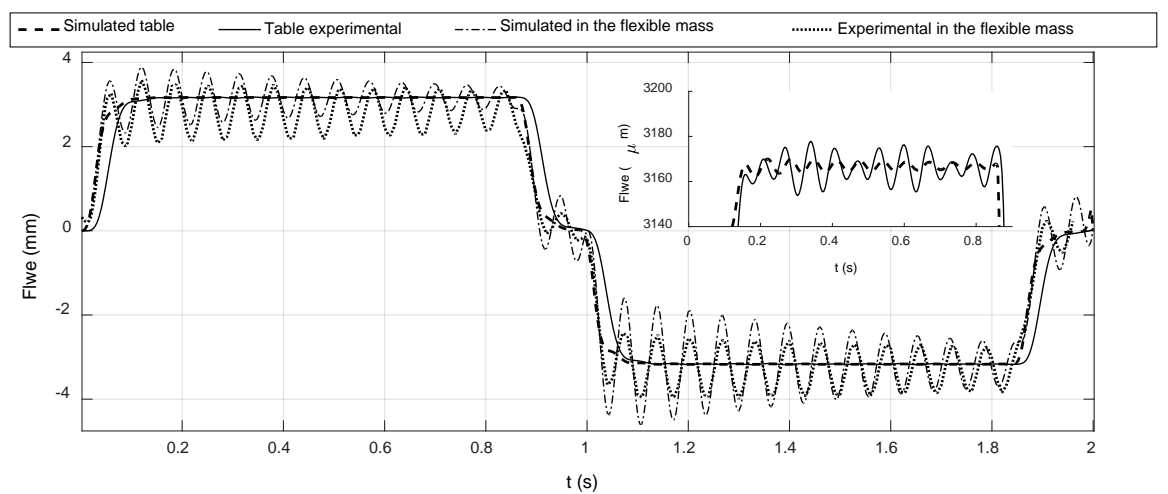

Fig. 6. Following error in the table and flexible mass. $V f=7000 \mathrm{~mm} / \mathrm{min}$ and $\Delta x=100 \mathrm{~mm}$.

Several values of the jerk of the motion profile have been tested also. The jerk in a square sine profile conditions the shape of the motion and the appearance of harmonics near the load natural frequency. Fig.7 shows the position overshoot in the mass for several values of the programmed jerk and a feed speed of $15 \mathrm{~m} / \mathrm{min}$, experimentally measured and modelled. Above $150 \mathrm{~m} / \mathrm{s}^{3}$, the overshoot tends to increase until it stabilizes in $0,8 \mathrm{~mm}$. Below $150 \mathrm{~m} / \mathrm{s}^{3}$, the curve has several "valleys", that is, there are values of the jerk that minimize the overshoot and could be considered as optimal, for example at $150 \mathrm{~m} / \mathrm{s}^{3}$. As a reference, machine tool manufacturers tend to be conservative with the jerk, and rarely increase it above $30 \mathrm{~m} / \mathrm{s}^{3}$ to avoid position overshoots.

On the other hand, to account for the influence of the control, in Fig. 7, the discontinuous line represents the position overshoot calculated with just a mechanical model of the system in open loop. It predicts higher overshoots than measured, probably because there is not a position control trying to compensate the error in the table position and thus in the load position.

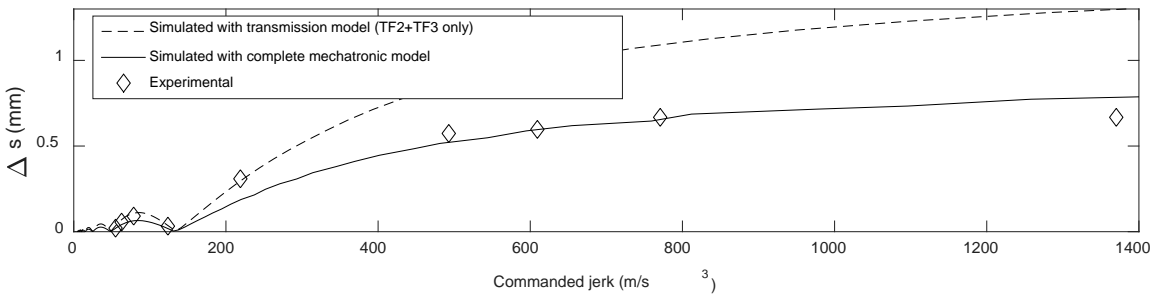

Fig. 7. Load overshoot $\Delta \mathrm{s}$ as a function of the programmed jerk, feed speed of $15 \mathrm{~m} / \mathrm{min}$. 


\section{Optimal jerk path planning}

The use of the graph in Fig. 7 is to enter with a predefined maximum overshoot and obtain the highest jerk, in order to have the most dynamic performance. What happens, is that at low enough jerk values, the predefined overshoot is never surpassed up to a limit, but then after that limit, there will appear several "valleys" in the graph, where with even higher values of the jerk and no overshoot. These isolated areas can be considered for an optimal dynamic performance.
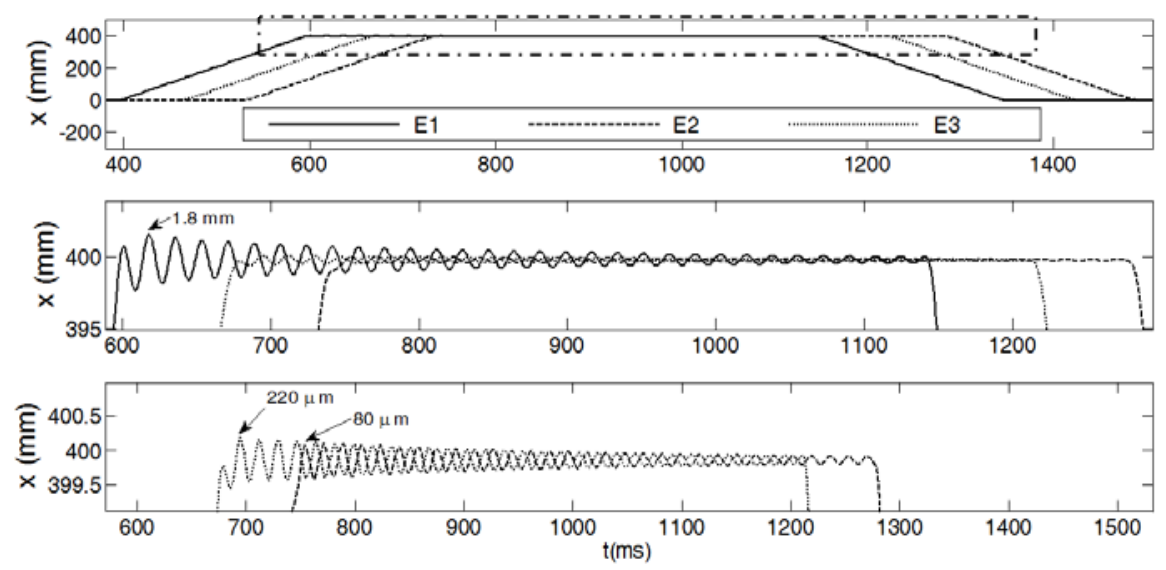

Fig. 8. Measured position in tests $E 1\left(j=846 \mathrm{~m} / \mathrm{s}^{3}\right), E 2\left(j=256 \mathrm{~m} / \mathrm{s}^{3}\right)$ and $E 3\left(\mathrm{j}=171 \mathrm{~m} / \mathrm{s}^{3}\right)$.

A detailed analysis in frequency domain of the square sine velocity profile reveals that these values happen when the acceleration time of the profile matches the relation with the natural frequency $f_{n}$ of Eq. 3 . Given the math of this profile, the optimal jerk as well as the corresponding acceleration can also be calculated as a function of the feed speed to program and the natural frequency as follows:

$$
t_{a c c}=\frac{2 n+1}{2} \frac{1}{f_{n}} \quad j=2 V_{f}\left(\frac{\pi f_{n}}{2 n+1}\right)^{2} \quad a=V_{f} \frac{\pi f_{n}}{2 n+1} \quad n=1,2,3, \ldots
$$

In Fig. 8, the measured position of the load in three experimental tests at 30 $\mathrm{m} / \mathrm{min}$ and a displacement of $400 \mathrm{~mm}$ is shown to prove this. E1 is a test at a jerk of $846 \mathrm{~m} / \mathrm{s}^{3}$, higher than the first valley and the overshoot reaches $1,8 \mathrm{~mm}$, in E2 the optimal jerk of $256 \mathrm{~m} / \mathrm{s}^{3}$ for $\mathrm{n}=1$ has been used and the overshoot falls to 80 $\mu \mathrm{m}$, and in $\mathrm{E} 3$ a lower jerk of $171 \mathrm{~m} / \mathrm{s}^{3}$ outside the valley has been tested and the overshoot rises again up to $220 \mu \mathrm{m}$. 


\section{Conclusions}

In this work it has been proved how the use of a mechatronic model that integrates the dynamics of the drive, transmission, compliant load and the control allows estimating the overshoot and vibration that will be present in the load. What is more, that model can be used to calculate the best values of the jerk and acceleration for a motion profile minimizing the overshoot at the end of the displacement. This approach has been tested successfully in a test bench if a compliant load with one predominant mode but can be applied to cases where the load has several modes or the modes of the load and the transmission chain are more similar.

Acknowledgments Authors acknowledge the financial support from the Spanish Government through the MINECO DPI2015-64450-R (MINECO/FEDER, UE) and the support through the project IT949-16, given by the Basque Government.

\section{References}

1. Dequidt, A., Castelain, J.-M., and Valdès, E., Mechanical pre-design of high performance motion servomechanisms, Mech. Mach. Theory, 35(8), 1047-1063, (2000)

2. Altintas, Y., Verl, A., Brecher, C., Uriarte, L., and Pritschow, G., Machine tool feed drives, CIRP Ann. - Manuf. Technol., 60(2), 779-796, (2011)

3. Caracciolo, R., and Richiedei, D., Optimal design of ball-screw driven servomechanisms through an integrated mechatronic approach, Mechatronics, 24(7), 819-832, (2014)

4. Neugebauer, R., Denkena, B., and Wegener, K., Mechatronic Systems for Machine Tools, CIRP Ann. - Manuf. Technol., 56(2), 657-686, (2007)

5. Wu, S.-T., Lian, S.-H., and Chen, S.-H., Vibration control of a flexible beam driven by a ballscrew stage with adaptive notch filters and a line enhancer, J. Sound Vib., 348, 71-87, (2015)

6. Fortunato, A., and Ascari, A., The virtual design of machining centers for HSM: Towards new integrated tools, Mechatronics, 23(3), 264-278, (2013) 